С.Н.Авдеев, Н.А.Кариевская, Г.Е.Баймаканова, А.В.Черняк

Годичное наблюдение за больными, перенесшими острое повреждение легких / острый респираторный дистресс синдром, вызванный вирусом гриппа A / H1N1

ФГУ "НИИ пульмонологии" ФМБА России: 105077, Москва, ул. 11-я Парковая, 32, корп. 4

\title{
S.N.Avdeev, N.A.Karchevskaya, G.E.Baimakanova, A.V.Cherniak \\ A one-year follow-up study of patients survived after ALI / ARDS caused by influenza A / H1N1
}

\begin{abstract}
Summary
The study analyzed long-term consequences of acute lung injury / acute respiratory distress syndrome (ALI / ARDS) caused by influenza A / H1N1 and effects of long-term treatment with N-acetylcysteine (NAC) on clinical and functional recovery of survived patients.

This was an open, prospective, 12-month study involved 22 patients survived from ALI / ARDS caused by influenza A / H1N1. The patients' lung function, arterial blood gases, physical tolerance in 6-min walking test (6MWT) and computed tomography (CT) of the lungs were monitored in 3, 6, and 12 months after discharge.

During the follow-up, all lung function parameters improved: FVC, TLC, and DL $\mathrm{CO}_{\mathrm{CO}}$ increased by $28.7 \%, 17.5 \%$ and $31.4 \%$, respectively $(p<0.05$ for all). In a year after discharge, $\mathrm{DL}_{\mathrm{CO}}<80 \%$ pred. was found in $23 \%$ of patients. At 12 months, the mean $6 \mathrm{MWT}$ distance increased from $454 \pm$ 37 to $568 \pm 47 \mathrm{~m}(p=0.002)$. Significant improvement was noted in CT findings and $41 \%$ of patients had quite normal lung CT at $12 \mathrm{months}$.

Therapy with NAC $(n=11)$ has led to more rapid improvement in $\mathrm{DL}_{\mathrm{co}}(76.3 \pm 11.8 \%$ pred. vs $63.0 \pm 10.6 \%$ pred. in controls; $p=0.012)$ and 6MWT distance ( $533 \pm 42 \mathrm{~m}$ vs $490 \pm 46 \mathrm{~m}$, respectively; $p=0.033)$ at 3 months.

Therefore, patients survived from ALI / ARDS caused by influenza A / H1N1 demonstrated significant recovery of lung function, gas exchange, lung $\mathrm{CT}$ picture and physical tolerance but in some of them, persistent disorders of lung function (mainly in $\mathrm{DL}_{\mathrm{CO}}$ ) and lung CT have been still found at 1 year after discharge. Treatment with NAC allowed more rapid improvement in DL $\mathrm{LO}_{\mathrm{Co}}$ and exercise tolerance.

Key words: $\mathrm{N}$-acetylcysteine, influenza, acute respiratory distress-syndrome.
\end{abstract}

\section{Резюме}

Задачей исследования было изучение долгосрочных последствий у больных, переживших острое повреждение легких / острый респираторный дистресс-синдром (ОПЛ / ОРДС), вызванный вирусом гриппа А / H1N1, а также анализ влияния длительной терапии N-ацетилцистеином - NAC (Флуимуцил, Zambon Group, Италия) на динамику клинических и функциональных изменений у данных пациентов.

Исследование длительностью 12 мес. имело проспективный, открытый дизайн. В него были включены 22 больных, перенесших ОПЛ / ОРДС, вызванный вирусом гриппа А / H1N1. Через 3, 6 и 12 мес. после выписки пациентам проводились измерение функции внешнего дыхания (ФВД), газовый анализ артериальной крови, 6-минутный шаговый тест (6-МШТ) и компьютерная томография (КТ) грудной клетки.

В течение 1-го года у больных наблюдалось улучшение всех параметров ФВД: прирост ФЖЕЛ, OEЛ, DLco К 12-му мес. - на 28,7; 17,5 и $31,4 \%$ соответственно (для всех $p<0,05$ ). Через 1 год после выписки $\mathrm{DL}_{\text {со }}<80 \%$ сохранялось у $23 \%$ больных. Средняя дистанция в 6-МШТ за 12 мес. возросла от $454 \pm 37$ до $568 \pm 47$ м $(p=0,002)$. За время наблюдения отмечена значительная положительная динамика KT-изменений легких, к 12-му мес. исследования у $41 \%$ больных КТ-картина легких была практически нормальной. Терапия NAC

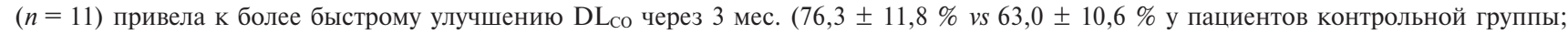
$p=0,012)$ и дистанции в 6-МШТ через 3 мес. (533 \pm 42 м $v s 490 \pm 46$ м; $p=0,033)$.

Таким образом, у пациентов, выживших после ОПЛ / ОРДС, вызванного вирусом гриппа А / H1N1, в течение 1-го года наблюдается значительная положительная динамика показателей ФВД, газообмена, КТ легких и физической выносливости, однако у ряда больных даже через 1 год после выписки сохраняются изменения ФВД (преимущественно DL $\mathrm{L}_{\mathrm{co}}$ и $\mathrm{KT}$ легких. Терапия NAC приводит к более быстрому улучшению $\mathrm{DL}_{\mathrm{co}}$ и приросту дистанции в нагрузочном тесте.

Ключевые слова: $\mathrm{N}$-ацетилцистеин, грипп, острый респираторный дистресс-синдром.

В 2009-2010 гг. мы стали свидетелями пандемии, вызванной вирусом гриппа А / H1N1 [1, 2]. Пандемический вирус гриппа А / H1N1 отличался по своей патогенности от сезонного гриппа по 2 ключевым аспектам: во-первых, вирусная инфекция гриппом A / H1N1 чаще всего поражала лиц более молодого возраста, а, во-вторых, вирус был способен инфицировать нижние дыхательные пути и вызвать быстро прогрессирующую пневмонию и острую дыхательную недостаточность (ОДН) [3]. В сезон пандемии гриппа A / H1N1 от 1 \% до 10 \% всех пациентов нуждались в госпитализации, и общая летальность больных составила 0,5 \% [4]. При анализе данных больных гриппом A / H1N1, находившихся в отделениях интенсивной терапии (ОРИТ) разных городов РФ и разных стран, обращало на себя внимание разительное сходство этих пациентов: молодой возраст (в среднем 30-40 лет), очень высокая потребность в искусственной вентиляции легких (ИВЛ) (70-100\%) и очень высокая летальность (17-50 \%) [5]. 
Среди пациентов, госпитализированных в ОРИТ, острое повреждение легких / острый респираторный дистресс-синдром (ОПЛ / ОРДС) был диагностирован у 10-56 \% больных [6-8]. Выживаемость пациентов с ОПЛ / ОРДС в последние годы улучшилась [9] однако по-прежнему не очень много известно о долгосрочных последствиях ОПЛ / ОРДС и возможностях терапии этой патологии. Пациенты, пережившие ОПЛ / ОРДС, характеризуются высоким риском развития органических и функциональныых осложнений, что может быть связано не только с повреждением паренхимы легких, но и с полиорганной недостаточностью и длительным периодом пребывания в ОРИТ. В нескольких работах изучались долгосрочные последствия ОПЛ / ОРДС на основе оценки функциональных легочных тестов, тестов с физической нагрузкой, нейрофизиологических и когнитивных исследований и оценки качества жизни, и в большинстве из них было показано, что изменения в этих сферах жизнедеятельности пациентов персистируют длительное время после их выписки из ОРИТ [10, 11$]$.

Относительно недавно появились исследования, посвященные долгосрочным последствиям ОПЛ / ОРДС, связанных с тяжелым острым респираторным синдромом [12-14], однако пока нет никаких данных о последствиях ОПЛ / ОРДС, вызванных пандемическим вирусом гриппа А / H1N1. Целью настоящего проспективного исследования явилось изучение долгосрочных последствий у больных, переживших ОПЛ / ОРДС, вызванный вирусом гриппа A / H1N1. Кроме того, был проведен анализ влияния длительной терапии $\mathrm{N}$-ацетилцистеином (NAC) на динамику клинических и функциональных изменений у данных пациентов.

\section{Материалы и методы}

Исследование носило проспективный, открытый наблюдательный характер, длительность исследования составила 12 мес. В исследование были включены пациенты, выписанные из стационаров Москвы и перенесшие в период с ноября 2009 г. по февраль 2010 г. ОПЛ / ОРДС, вызванный вирусом гриппа А / H1N1. Последний пациент присоединился к исследованию в феврале 2010 г.

Критериями включения пациентов в исследование были: вирусная инфекция гриппом A / H1N1, документированный диагноз ОПЛ / ОРДС; возраст $>18$ лет. ОПЛ / ОРДС диагностировали в соответствии с критериями, рекомендованными Американо-Европейской согласительной конференцией по ОПЛ / ОРДС (1994): острое начало; 2-сторонние инфильтраты на рентгенограмме органов грудной клетки; гипоксемический индекс $\left(\mathrm{PaO}_{2} / \mathrm{FiO}_{2}\right)-$ $<300$ мм рт. ст.; отсутствие нескорригированной левожелудочковой недостаточности или давление заклинивания в легочной артерии $<18$ мм рт. ст. [15]. Инфекция гриппа A / H1N1 была подтверждена во всех случаях обнаружением РНК вируса гриппа А / H1N1 с помощью полимеразной цепной реакции с обратной транскриптазой в режиме реального времени.

Критерии исключения больных из исследования были следующими: патология, имитирующая ОПЛ / ОРДС (застойная сердечная недостаточность, интерстициальные легочные заболевания и др.); возраст моложе 18 лет; тяжелая печеночная недостаточность, почечная недостаточность, злокачественные новообразования.

Тяжесть состояния больных во время острой фазы инфекции оценивалась по параметрам гипоксемического индекса $\mathrm{PaO}_{2} / \mathrm{FiO}_{2}$ (минимальные значения в истории заболевания), по шкале АРАСНЕ II (максимальные значения в истории заболевания) [16] и по шкале полиорганной недостаточности MODS [17].

Больных обследовали в амбулаторных условиях через 3, 6 и 12 мес. после выписки из стационара. Во время каждого визита пациентам выполняли исследование функции внешнего дыхания (ФВД), общий анализ крови, газовый анализ артериальной крови, 6-минутный шаговый тест (6-МШТ), измерение насыщения крови кислородом по данным пульсоксиметрии $\left(\mathrm{SpO}_{2}\right)$ в покое и на высоте физической нагрузки, оценивали одышку по шкалам MRC и Borg, а также проводили компьютерную томографию (KT) грудной клетки (через 6 и 12 мес.).

Исследование ФВД проводилось путем анализа кривой "поток-объем", показателей спирометрии, общей плетизмографии, диффузионного теста с использованием оборудования и программного обеспечения Master Screen Body (Jaeger, Германия). Bce измерения объемов регистрировались в системе BTPS (BT $-\mathrm{t}^{\circ}$ тела, P - окружающее атмосферное давление, S - полное насыщение водяными парами). При анализе ФВД использовались следующие показатели: форсированная жизненная емкость легких (ФЖЕЛ), объем форсированного выдоха за 1-ю с $\left(\mathrm{O}^{\circ} \mathrm{B}_{1}\right)$, отношение ОФВ легких (ОЕЛ), диффузионная способность легких $\left(\mathrm{DL}_{\mathrm{CO}}\right)$. Оценку полученных результатов проводили при сопоставлении данных с должными величинами, рассчитанные по формулам Европейского сообщества стали и угля 1993 г. [18, 19].

Показатели газового состава крови и кислотноосновного состояния определяли с помощью с помощью автоматического газоанализатора ABL 750 (Radiometer, Швеция). Регистрировали следующие показатели: парциальное напряжение $\mathrm{O}_{2}$ в артериальной крови $\left(\mathrm{PaO}_{2}\right)$ и парциальное напряжение $\mathrm{CO}_{2}$ в артериальной крови $\left(\mathrm{PaCO}_{2}\right)$.

Количественная оценка одышки у больных проводилась во время повседневной активности по шкале MRC (Medical Research Council) [20] и во время физической нагрузки - по шкале Borg [21].

6-МШТ выполняли в соответствии со стандартными протоколами [22]. Пациенты были проинструктированы о целях теста, им было предложено ходить по измеренному коридору в своем собственном темпе, стараясь преодолеть максимальное расстояние в течение 6 мин. Пациентам было разрешено останавливаться и отдыхать во время теста, 
однако они должны были возобновить ходьбу, когда они сочтут это возможным. Во время ходьбы разрешалось подбадривать больных фразами "Все идет хорошо", "Продолжайте в том же темпе". Перед началом и в конце теста оценивались $\mathrm{SpO}_{2}$ и диспноэ по шкале Borg [22].

КТ легких проводилось на спиральном компьютерном томографе SOMATOM Emotion (General Electric, США). Использовался алгоритм высокого разрешения. Высокоразрешающая КТ имела следующие физические параметры: 140 кВ / 240 мА, время сканирования - 2 с, толщина среза - 10 мм, высокоразрешающий (костный) алгоритм реконструкции, прицельная реконструкция измененного участка легочной ткани с ограничением зоны интереса, основное окно (уровень / ширина) - 700 / 1000 HU.

Кроме того, анализировали влияние терапии NAC на динамику клинических и функциональных изменений после перенесенного ОПЛ / ОРДС. 11 пациентов получали NAC (Флуимуцил, Zambon Group, Италия) в дозе 1800 мг в сутки per os в течение 3 мес. Их клинико-функциональные параметры сравнивали с показателями больных, не получавших данной терапии.

Все численные данные представлены как mean \pm $S D$ (или medain). Для оценки различий множественных одноименных показателей между группами использовался тест ANOVA. Достоверность различий количественных показателей между несвязанными группами определялись при помощи непарного t-критерия Стьюдента и U-теста Манна-Уитни (при ненормальном распределении). Различия считались статистически достоверными при $p<0,05$. Статистическая обработка результатов была проведена при помощи программных пакетов Statistica for Windows 6.0. и SPSS for Windows 13.

\section{Результаты}

\section{Характеристика больных}

В исследовании участвовали 22 больных, перенесших ОПЛ / ОРДС, вызванный вирусом гриппа А / H1N1. Средний возраст пациентов составил $42 \pm 12$ лет, лишь небольшая доля из них были курильщиками (23\%) и имели хронические сопутствующие заболевания. 4 пациентки на момент развития гриппа А / H1N1 были беременными (табл. 1). Большинство пациентов во время ОПЛ / ОРДС получали ИВЛ (54\%) или неинвазивную вентиляцию легких - НВЛ (18\%). Средние показатели тяжести больных по шкале АРАСНЕ II в острой фазе заболевания составляли $20,3 \pm 2,4$ балла. Терапию противовирусными препаратами и глюкокортикостероидами (ГКС) получали большинство пациентов - 86 \% и $68 \%$ соответственно (табл. 1). Средняя длительность госпитализации пациентов в ОРИТ составила 19,4 \pm 10,1 дня, а всего больные провели в стационаре $31,7 \pm 12,6$ дня.

\section{Изменение функциональных легочных параметров}

Исходно, т. е. после выписки из стационара, у большинства больных были выявлены рестриктивные изменения ФВД (средние значения ФЖЕЛ - 82,3 \% и ОЕЛ $(92,7 \%)$ и выраженное снижение диффузионной способности легких (средняя $\mathrm{DL}_{\mathrm{co}}(50,9 \%)$ (табл. 2). С течением времени наблюдалось улучшение функциональных показателей: к 3-му мес. ФЖЕЛ и ОЕЛ выросли на 26,6 \% и 15,8 \% соответственно, к 6-му мес. - на 26,8 \% и 17,1 \% и к 12-му мес. - на 28,7 \% и 17,5\% ( $p<0,05)$. Также была зарегистрирована положительная динамика со стороны $\mathrm{DL}_{\mathrm{Co}}$ : через 3 мес. выявлен прирост показателя на $28,8 \%$, через 6 мес. - на 29,7\% и через 12 мес. - на $31,4 \%$. Таким образом, через год после выписки пациентов из стационара рестриктивный дефект (ФЖЕЛ < 80 \% или ОЕЛ < $80 \%$ ) сохранялся у $9 \%$ больных, обструктивный дефект $\left(\mathrm{OФB}_{1}<80 \%\right.$ и ОФВ 1 ФЖЕЛ < $70 \%$ ) - у $5 \%$, и у $23 \%$ пациентов наблюдалось значимое снижение диффузионной способности легких (DL $\left.\mathrm{DL}_{\mathrm{CO}}<80 \%\right)$.

На момент выписки большинство пациентов имели практически нормальные показатели газов артериальной крови (средние значения $\mathrm{PaO}_{2}-74,1$ мм рт. ст. и $\mathrm{PaCO}_{2}-38,4$ мм рт. ст.) (табл. 2). Исходно снижение $\mathrm{SpO}_{2}<90 \%$ в покое и во время нагрузочного теста имели лишь 1 (5\%) и 2 (9\%) больных соответственно, однако уже к 6-му мес. исследования десатурация не обнаруживалась ни в одном случае.

\section{Изменение одышки и параметров физической выносливости}

При выписке из стационара большинство пациентов предъявляли жалобы на одышку в повседневной жизни (число баллов по шкале $\mathrm{MRC}-\geq 1$ ) и во время

Таблица 1

Исходные показатели выживших больных $(n=22)$ с ОПЛ / ОРДС, вызванным вирусом гриппа A / H1N1

\begin{tabular}{|c|c|}
\hline Параметры & Значения \\
\hline Возраст, лет & $42,2 \pm 12,4$ \\
\hline Пол, м / ж & $12 / 10$ \\
\hline ИМТ, кг / м² & $31,9 \pm 18,2$ \\
\hline Курильщики & $5(23 \%)$ \\
\hline \multicolumn{2}{|c|}{ Сопутствующие заболевания } \\
\hline Морбидное ожирение (ИМТ $\geq 40$ кг / м²) & $1(5 \%)$ \\
\hline ХОБЛ и / или БА & $2(9 \%)$ \\
\hline ИБС и / или ГБ & $2(9 \%)$ \\
\hline Сахарный диабет & $1(5 \%)$ \\
\hline Беременность & $4(18 \%)$ \\
\hline \multicolumn{2}{|l|}{ Терапия } \\
\hline $\begin{array}{l}\text { противовирусная (осельтамивир, ингавирин) } \\
\text { системные ГКС }\end{array}$ & $\begin{array}{l}19(86 \%) \\
15(68 \%)\end{array}$ \\
\hline Шкала APACHE II, баллы (max) & $20,3 \pm 2,4$ \\
\hline Шкала MODS, баллы (тах) & $6,0 \pm 1,5$ \\
\hline $\mathrm{PaO}_{2} / \mathrm{FiO}_{2}$, мм рт. ст. (min) & $145,6 \pm 44,1$ \\
\hline ИВЛ & $12(54 \%)$ \\
\hline Трахеостомия & $9(41 \%)$ \\
\hline Длительность ИВЛ & $16,3 \pm 8,1$ \\
\hline НВЛ & $4(18 \%)$ \\
\hline Длительность пребывания в ОРИТ & $19,4 \pm 10,1$ \\
\hline Длительность пребывания в стационаре & $31,7 \pm 12,6$ \\
\hline
\end{tabular}

Примечание: ИМТ - индекс массы тела, ХОБЛ - хроническая обструктивная болезнь легких, БА - бронхиальная астма, ИБС - ишемическая болезнь сердца, ГБ - гипертоническая болезнь. 
Изменение функцинальных параметров в течение 12 мес. у выживиих больных с ОПЛ / ОРДС, вызванным вирусом гриппа A / H1N1

\begin{tabular}{|c|c|c|c|c|c|}
\hline \multirow[t]{2}{*}{ Параметры } & \multicolumn{4}{|c|}{ Значения (mean $\pm S D)$} & \multirow[t]{2}{*}{$p$} \\
\hline & исходно & 3 мес. & 6 мес. & 12 мес. & \\
\hline РаO & $74,1 \pm 10,6$ & $81,6 \pm 12,4$ & $84,3 \pm 12,1$ & $86,4 \pm 11,5$ & 0,030 \\
\hline $\mathrm{PaCO}_{2}$, мм рт. ст. & $38,4 \pm 13,1$ & $38,6 \pm 13,2$ & $38,8 \pm 16,2$ & $38,5 \pm 13,4$ & 0,048 \\
\hline $\mathrm{SpO}_{2}, \%$ (в покое) & $95,8 \pm 1,1$ & $96,2 \pm 1,1$ & $96,2 \pm 1,1$ & $97,45 \pm 1,2$ & 0,005 \\
\hline ОФВ $1, \%_{\text {долж. }}$ & $81,4 \pm 30,8$ & $105,4 \pm 18,4$ & $107,4 \pm 15,9$ & $110,7 \pm 14,8$ & 0,016 \\
\hline ФЖЕЛ, \% & $82,3 \pm 28,8$ & $108,9 \pm 22,9$ & $109,1 \pm 21,3$ & $113,4 \pm 18,3$ & 0,029 \\
\hline ОЕЛ, \% & $92,7 \pm 21,0$ & $108,5 \pm 17,8$ & $109,8 \pm 15,7$ & $110,3 \pm 14,5$ & 0,006 \\
\hline $\mathrm{DL}_{\mathrm{co}} \mathrm{SB}, \%_{\text {долж. }}$ & $50,9 \pm 20,9$ & $69,7 \pm 11,3$ & $80,6 \pm 13,5$ & $82,3 \pm 14,8$ & 0,001 \\
\hline Дистанция 6-МШт, м & $453,7 \pm 36,6$ & $511,2 \pm 44,0$ & $554,5 \pm 57,6$ & $567,5 \pm 46,9$ & 0,002 \\
\hline Одышка по шкале MRC (в повседневной жизни), баллы & $1,1 \pm 0,6$ & $0,4 \pm 0,5$ & $0,2 \pm 0,4$ & $0,1 \pm 0,3$ & 0,003 \\
\hline Одышка по шкале Borg (на высоте нагрузки), баллы & $1,8 \pm 1,4$ & $1,2 \pm 0,6$ & $0,7 \pm 0,8$ & $0,4 \pm 0,6$ & 0,001 \\
\hline $\mathrm{SpO}_{2}$ (на высоте нагрузки), \% & $93,6 \pm 2,9$ & $95,8 \pm 1,8$ & $96,1 \pm 1,3$ & $96,2 \pm 1,1$ & 0,063 \\
\hline
\end{tabular}

нагрузочного теста (число баллов по шкале Borg $\geq 1)-64 \%$ и $91 \%$ больных соответственно. Однако на протяжении 12 мес. одышка уменьшилась и полностью исчезла у подавляющего большинства пациентов (табл. 2). К 12-му мес. наблюдения одышка при повседневной активности сохранялась лишь у 1 (5\%) больного, а одышка во время физической нагрузки - у 5 (23\%) больных. Толерантность к физическим нагрузкам также претерпела значительную положительную динамику. Исходно средняя дистанция в 6-МШТ составляла 454 м, однако в течение всего периода исследования данный показатель постепенно возрастал (табл. 2), и к 12-му мес. он составлял в среднем 568 м $(p=002)$.

\section{Динамика изменений на основании КТ легких}

После выписки из стационара изменения картины КТ легких были выявлены у всех больных, перенесших ОПЛ / ОРДС (табл. 3). Чаще всего выявляли изменения по типу "матового стекла" (59\%), мультифокальный фиброз с изменениями по типу "матового стекла" (41\%), уплотнение междолькового интерстиция (36 \%), консолидаты (32\%) и тракционные бронхоэктазы (32 \%) и др. Указанные изменения имели преимущественно перибронховаскулярное или суб- плевральное распространение в нижних и средних зонах легких. За время наблюдения отмечена значительная положительная динамика КТ-изменений легких, к 6-му мес. исследования у 7 (32\%) больных картина КТ легких была практически нормальной, а к 12 мес. - у 9 (41 \%) пациентов (табл. 3). Примеры положительной динамики КТ-изменений у пациентов представлены на рис. 1 и 2.

\section{Социальная адаптация пациентов}

На момент включения в исследование потребность в постоянном постороннем уходе возникала у 3 (14\%) пациентов, через 3 мес. - у 2 (9\%) больных, а через 6 и 12 мес. - у 1 (5 \%) пациента. В длительной кислородотерапии в домашних условиях (с помощью концентратора кислорода) исходно нуждался лишь 1 больной, однако вследствие положительной динамики его статуса газообмена к 6-му мес. стало возможным прекратить кислородотерапию. После выписки из стационара трахеостома была выполнена лишь одной пациентке вследствие развития посттрахеостомического стеноза трахеи. В ближайший месяц после выписки из стационара к работе приступили $46 \%$ пациентов, через 3 мес. - $82 \%$, через 12 мес. - $82 \%$ больных.

Таблица 3

Изменение КТ-параметров в течение 12 мес. у выживших больных с ОПЛ / ОРДС, вызванным вирусом гриппа A / H1N1

\begin{tabular}{|c|c|c|c|}
\hline \multirow[t]{2}{*}{ КТ-изменения } & \multicolumn{3}{|c|}{ Значения (n, \%) } \\
\hline & исходно & 6 мес. & 12 мес. \\
\hline Изменения по типу "матового стекла" & $13(59)$ & $7(32)$ & $6(27)$ \\
\hline Двусторонний базальный фиброз с изменениями по типу "матового стекла" & $4(18)$ & 2 (9) & 2 (9) \\
\hline Локальный фиброз & $7(32)$ & $5(23)$ & $5(23)$ \\
\hline Мультифокальный фиброз & $6(27)$ & $4(18)$ & $4(18)$ \\
\hline Мультифокальный фиброз с изменениями по типу "матового стекла" & $9(41)$ & $5(23)$ & $4(18)$ \\
\hline Тракционные бронхоэктазы & $7(32)$ & $3(14)$ & $3(14)$ \\
\hline Консолидаты & $7(32)$ & 0 & 0 \\
\hline "Сотовые" изменения & $2(9)$ & $2(9)$ & $2(9)$ \\
\hline Уплотнение междолькового интерстиция & $8(36)$ & $5(23)$ & $4(18)$ \\
\hline Пневмоцеле & $2(9)$ & $1(5)$ & $1(5)$ \\
\hline Норма & 0 & $7(32)$ & $9(41)$ \\
\hline
\end{tabular}




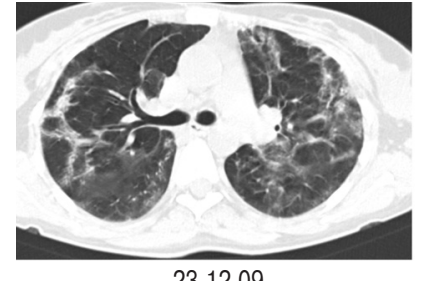

23.12 .09

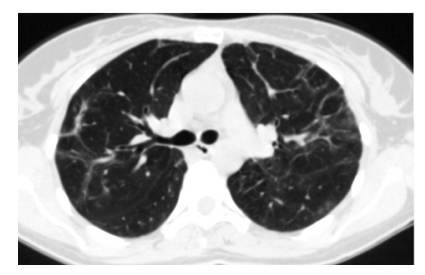

11.01.11

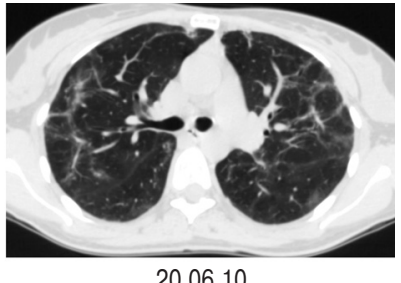

20.06.10

Рис. 1. Динамика КТ-картины больной Г. (представлена серия КТ: при выписке из стационара, через 6 и через 12,5 мес.)

Влияние терапии NAC на клинико-функциональные параметры

Было проведено сравнение динамики изменений клинических и функциональных показателей у пациентов, принимавших NAC $(n=11)$ и не получав-

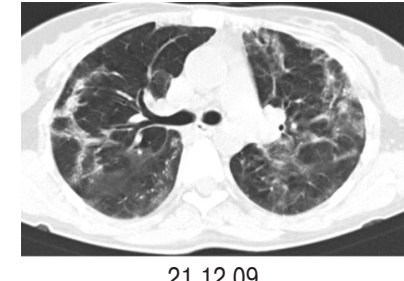

21.12 .09

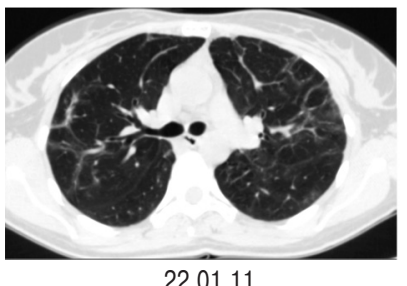

ших данный препарат $(n=11)$. Больные 2 групп сравнения практически не отличались по параметрам газообмена, ФВД, одышки и нагрузочного теста (табл. 4). Достоверные отличия между группами терапии были выявлены при сравнении показателей

Таблица 4

Различия по функциональным параметрам в течение 12 мес у выживших больных с ОПЛ / ОРДС, вызванным вирусом гриппа A / H1N1, в зависимости от назначения NAC

\begin{tabular}{|c|c|c|c|c|}
\hline \multirow[t]{2}{*}{ Параметры } & \multicolumn{4}{|c|}{ Значения (mean \pm SD) } \\
\hline & исходно & 3 мес. & 6 мес. & 12 мес. \\
\hline $\mathrm{PaO}_{2}$, мм рт. ст. (+ NAC) & $73,3 \pm 12,2$ & $82,8 \pm 11,3$ & $84,8 \pm 12,5$ & $85,6 \pm 11,8$ \\
\hline $\mathrm{PaO}_{2}$, мм рт. ст. (- NAC) & $74,8 \pm 10,3$ & $79,0 \pm 13,2$ & $83,6 \pm 10,2$ & $86,8 \pm 11,3$ \\
\hline р между группами & 0,759 & 0,477 & 0,808 & 0,810 \\
\hline $\mathrm{PaCO}_{2}$, мм рт. ст. (+ NAC) & $39,4 \pm 12,1$ & $39,4 \pm 12,6$ & $39,3 \pm 14,1$ & $39,2 \pm 11,4$ \\
\hline $\mathrm{PaCO}_{2}$, мм рт. ст. (- NAC) & $37,3 \pm 15,1$ & $37,4 \pm 14,1$ & $38,4 \pm 22,1$ & $37,3 \pm 15,2$ \\
\hline р между группами & 0,723 & 0,729 & 0,901 & 0,744 \\
\hline $\mathrm{SpO}_{2}, \%$ (в покое) (+ NAC) & $96,0 \pm 1,1$ & $96,6 \pm 1,2$ & $96,6 \pm 1,0$ & $97,6 \pm 1,5$ \\
\hline $\mathrm{SpO}_{2}, \%$ (в покое) (- NAC) & $95,4 \pm 1,2$ & $95,5 \pm 1,4$ & $96,5 \pm 1,3$ & $97,0 \pm 0,9$ \\
\hline р между группами & 0,236 & 0,062 & 0,842 & 0,269 \\
\hline OФB ${ }_{1}, \%_{\text {долж. }}(+\mathrm{NAC})$ & $71,6 \pm 18,9$ & $109,4 \pm 23,3$ & $111,7 \pm 20,4$ & $113,8 \pm 19,1$ \\
\hline ОФВ ${ }_{1}, \%_{\text {долж. }}(-\mathrm{NAC})$ & $88,1 \pm 31,4$ & $101,3 \pm 13,2$ & $103,2 \pm 10,3$ & $107,6 \pm 10,3$ \\
\hline р между группами & 0,151 & 0,328 & 0,232 & 0,355 \\
\hline ФЖЕЛ, \% \%олж. (+ NAC) & $75,9 \pm 26,6$ & $111,2 \pm 27,1$ & $114,76 \pm 26,4$ & $117,3 \pm 21,9$ \\
\hline ФЖЕЛ, \% & $92,6 \pm 30,3$ & $104,5 \pm 19,9$ & $107,4 \pm 17,1$ & $109,4 \pm 15,3$ \\
\hline р между группами & 0,185 & 0,516 & 0,678 & 0,338 \\
\hline ОЕЛ, \% ${ }_{\text {долж. (+ NAC) }}$ & $87,0 \pm 17,3$ & $110,2 \pm 23,3$ & $111,5 \pm 20,5$ & $112,4 \pm 19,4$ \\
\hline ОЕЛ, \% долж. $_{\text {(- NAC) }}$ & $98,3 \pm 26,7$ & $106,8 \pm 12,6$ & $107,9 \pm 11,2$ & $108,2 \pm 10,3$ \\
\hline р между группами & 0,253 & 0,675 & 0,615 & 0,533 \\
\hline $\mathrm{DL}_{\mathrm{co}} \mathrm{SB}, \%_{\text {долж. }}(+\mathrm{NAC})$ & $43,6 \pm 22,2$ & $76,3 \pm 11,8$ & $81,2 \pm 13,2$ & $84,7 \pm 13,9$ \\
\hline $\mathrm{DL}_{\mathrm{co}} \mathrm{SB}, \%_{\text {долж. }}(-\mathrm{NAC})$ & $57,9 \pm 20,7$ & $63,0 \pm 10,6$ & $80,0 \pm 14,5$ & $81,0 \pm 16,7$ \\
\hline р между группами & 0,134 & 0,012 & 0,841 & 0,579 \\
\hline Дистанция 6-Мшт, м (+ NAC) & $445 \pm 43$ & $533 \pm 42$ & $566 \pm 69$ & $578 \pm 58$ \\
\hline Дистанция 6-Мшт, м (-NAC) & $460 \pm 36$ & $490 \pm 46$ & $543 \pm 48$ & $557 \pm 36$ \\
\hline р между группами & 0,386 & 0,033 & 0,375 & 0,320 \\
\hline $\begin{array}{l}\text { Одышка по шкале Borg (на высоте нагрузки), } \\
\text { баллы (+ NAC) }\end{array}$ & $2,4 \pm 2,1$ & $1,0 \pm 0,5$ & $0,4 \pm 0,4$ & $0,1 \pm 0,8$ \\
\hline $\begin{array}{l}\text { Одышка по шкале Borg (на высоте нагрузки), } \\
\text { баллы (- NAC) }\end{array}$ & $1,4 \pm 0,5$ & $1,1 \pm 0,7$ & $0,9 \pm 1,1$ & $0,5 \pm 0,5$ \\
\hline р между группами & 0,140 & 0,704 & 0,172 & 0,175 \\
\hline $\mathrm{SpO}_{2}$ (на высоте нагрузки), \% (+ NAC) & $93,0 \pm 2,9$ & $95,6 \pm 1,1$ & $95,8 \pm 1,1$ & $96,0 \pm 0,7$ \\
\hline $\mathrm{SpO}_{2}$ (на высоте нагрузки), \% (- NAC) & $94,2 \pm 3,0$ & $96,0 \pm 2,5$ & $96,4 \pm 1,5$ & $96,4 \pm 1,5$ \\
\hline р между группами & 0,352 & 0,632 & 0,297 & 0,432 \\
\hline
\end{tabular}

Примечание: (+ NAC) - пациенты, принимавшие NAC, (- NAC) - пациенты, не принимавшие NAC. 


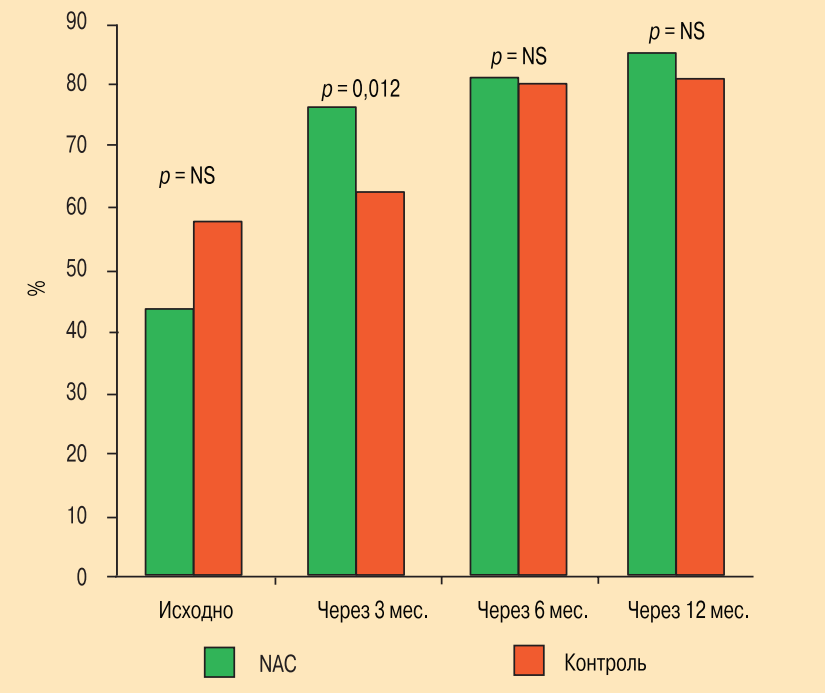

Рис. 3. Изменение DLco $(\%$ долж.) в течение 12 мес. у выживших больных с ОПЛ / ОРДС, вызванным вирусом гриппа А / H1N1, в зависимости от назначения NAC

диффузионной способности. Через 3 мес. DLсо у пациентов, принимавших NAC, составляла в среднем $76,3 \pm 11,8 \%$, а у пациентов контрольной группы $63,0 \pm 10,6 \%(p=0,012)$, хотя следует отметить, что исходно показатели $\mathrm{DL}_{\mathrm{co}}$ были немного ниже в группе терапии NAC (рис. 3). Также были отмечены достоверные различия между группами терапии по пройденной дистанции в 6-МШТ: через 3 мес. наблюдения данный показатель достиг $533 \pm 42$ м и $490 \pm 46$ м у пациентов, принимавших и не принимавших NAC ( $p=0,033)$ соответственно (рис. 4). В дальнейшем, через 6 и 12 мес., статистически достоверных различий по DL ${ }_{\mathrm{Co}}$ и дистанции в 6-МШТ выявлено не было, хотя данные параметры были все еще выше у пациентов, принимавших NAC.

\section{Обсуждение}

Проведенное исследование продемонстрировало, что у пациентов, перенесших ОПЛ / ОРДС, вызванный вирусом гриппа A / H1N1, в течение ближайшего года наблюдалась значительная положительная динамика показателей ФВД, газообмена, КТ легких и физической выносливости. Однако у некоторых больных даже через 1 год после выписки из стационара сохраняются изменения функциональных показателей (преимущественно $\mathrm{DL}_{\mathrm{co}}$ ) и $\mathrm{KT}$ легких. К сожалению, в настоящее время представленные данные о долгосрочной динамике клинико-функциональных параметров после ОПЛ / ОРДС, вызванного вирусом гриппа А / H1N1, являются единственными в своем роде, и их невозможно сопоставить с показателями пациентов из других клинических центров. Поэтому для сравнения стоит привести опубликованные данные о динамике функциональных показателей у больных, которые выжили после ОПЛ / ОРДС, вызванным другими причинами.

M.S.Herridge et al. в течение 1-го года проводили наблюдение за 109 пациентами, выжившими после ОПЛ / ОРДС (основными причинами которого бы-

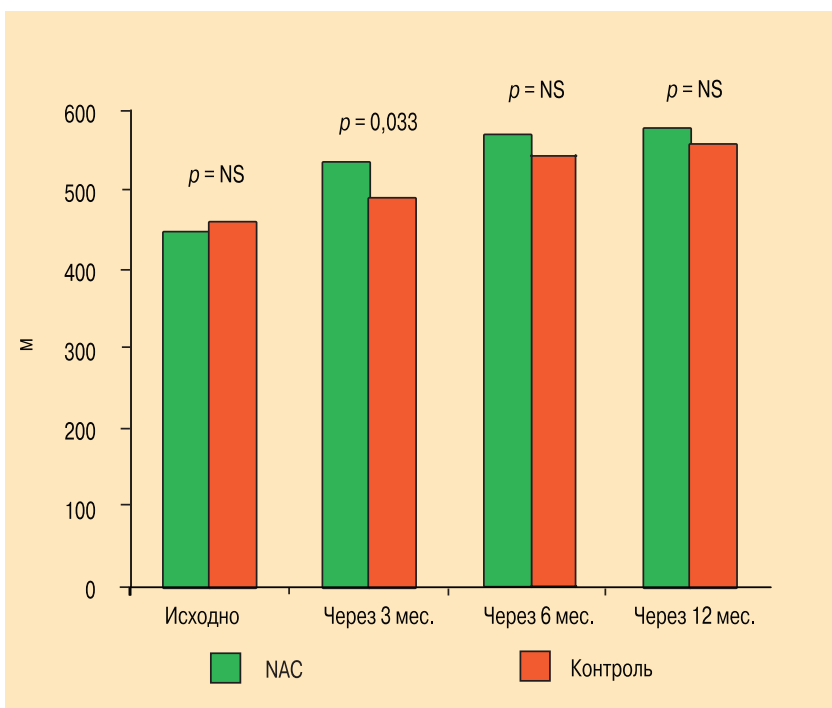

Рис. 4. Изменение дистанции в 6-МШТ в течение 12 мес. у выживших больных с ОПЛ / ОРДС, вызванным вирусом гриппа А / H1N1, в зависимости от назначения NAC

ли тяжелая пневмония и сепсис) [10]. Авторы отметили выраженное улучшение всех параметров ФВД в период наблюдения, однако даже к концу 1-го года средние значения DLсо отличались от нормальных (в среднем $72 \%$ через 12 мес.), ФЖЕЛ и ОФВ 1 также были достоверно ниже должных значений (в среднем $85 \%$ и $86 \%$ соответственно). Дистанция в 6-МШТ также выросла, но оставалась ниже должных величин (медиана - 422 м через 12 мес.). В исследовании J.Orme et al. проанализированы изменения функции легких у 66 пациентов, выживших после ОПЛ / ОРДС [11]. Оказалось, что через 1 год после выписки нарушение DL давляющего большинства пациентов - в 80 \% случаев, при этом ухудшение было легким в $46 \%$ случаев и умеренным - в $23 \%$. Рестриктивные и обструктивные нарушения ФВД обнаружили у 20 \% пациентов, обструктивный синдром чаще всего наблюдался у курильщиков. В другом исследовании, посвященном качеству жизни, связанному со здоровьем, у 73 пациентов, переживших ОПЛ / ОРДС, через 1 год после выписки больных из стационара большинство из них имели значимые респираторные симптомы, которые оказывали большое влияние на общее состояние пациентов [23].

Относительно недавно медицинский мир приобрел опыт борьбы с другим тяжелым вирусным заболеванием, основными проявлениями которого являлись ОДН и развитие ОПЛ / ОРДС, - тяжелым острым респираторным синдромом (severe acute respiratory syndrome - SARS) [24, 25]. В нескольких наблюдательных исследованиях уже проанализированы долгосрочные исходы пациентов после SARS [12-14].

C.M.Tansey et al. представили результаты 12-месячного проспективного наблюдения за 117 больными, выжившими после эпизода SARS в Торонто [12]. Снижение пройденной дистанции в 6-МШТ было выявлено у $31 \%$ больных через 3 мес. после выписки из стационара и у $18 \%$ - через 12 мес. Рестриктивные нарушения ФВД сохранялись у пациентов, 
выписанных из ОРИТ, через 3 и 6 мес. наблюдения, но к 12-му мес. у большинства больных функциональные легочные показатели вернулись к нормальным значениям, и лишь у 1 человека умеренный рестриктивный дефект ФВД сохранялся более 1 года. Проспективное когортное исследование D.S.Hui et al. показало, что среди 110 больных, выживших после ОПЛ / ОРДС, вызванного SARS, через 1 год после начала заболевания снижение DLсо сохранялось у $24 \%$ пациентов, а рентгенологические изменения - у $28 \%$ [26]. В целом серийные исследования физической выносливости больных с использованием 6-МШТ продемонстрировали значительное увеличение пройденной дистанции на протяжении 12 мес. наблюдения, однако физическая выносливость и качество жизни больных через 1 год после эпизода SARS все еще были достоверно хуже, чем у лиц здоровой группы контроля того же возраста.

Среди когорты 44 пациентов из Сингапура, переживших SARS, у $41 \%$ через 3 мес. после выписки из стационара сохранялось снижение физической выносливости, которое нельзя было объяснить только легочными функциональными нарушениями [27]. Снижение переносимости физических нагрузок у данных больных может быть связано не только с легочными функциональными параметрами, но также с экстралегочными факторами, такими как атрофия скелетных мышц (от бездействия), стероидиндуцированная и вирусная миопатия, полинейропатия / миопатия критических состояний [28], и с другими физиологическими причинами. Эти данные, конечно, отличаются от результатов настоящего годичного наблюдения. Возможно, одной из причин более благополучной картины физической выносливости в рассматриваемом случае является менее выраженная картина экстралегочной патологии. Так, в описываемом с этой статье исследовании полиорганная недостаточность встречалась реже, чем у больных ОПЛ / ОРДС в приведенных выше работах: в них оценка пациентов по шкале MODS coставляла 11 баллов [29], а у больных в настоящем исследовании $-\sim 6$ баллов.

В представленном наблюдении в течение 1-го года наблюдения у пациентов значительно улучшилась и даже полностью нормализовалась картина КТ легких. Интересно отметить, что обратному развитию подверглись даже те изменения, которые исходно расценивались как "фиброз" легких. В работе Y.-C.Chang et al. было показано, что, приблизительно через 5,5 мес. после эпизода SARS KT-изменения сохранялись у большинства пациентов (синдром "матового стекла" - в $70 \%$, ретикулярные изменения - в $50 \%$ случаев) [30]. В уже упоминавшемся исследовании M.S.Herridge et al. через 12 мес. после выписки рентгенограммы грудной клетки выглядели нормальными у 80 \% пациентов, перенесших ОПЛ / ОРДС [10].

Пока невозможно ответить на вопрос, какое время необходимо для полного восстановления функциональных и КТ-параметров и вероятно ли их полное восстановление в каждом конкретном случае.
Безусловно, для этого требуется более длительное наблюдение. Хотя, по данным единственного на сегодня в литературе опыта 5-летнего наблюдения за больными после ОПЛ / ОРДС, связанного с разными причинами, параметры ФВД, газообмена и физической выносливости через 1 год после выписки из стационара практически не претерпевают дальнейших положительных сдвигов в последующие 2-5 лет [29].

У большинства больных после эпизода ОПЛ / ОРДС имеются выраженные повреждения паренхимы легких, которые со временем трансформируются в легочный фиброз [9]. Однако, несмотря на эти опасения, никаких официальных рекомендаций по терапии, способной воспрепятствовать развитию фиброза легких после перенесенного ОПЛ / ОРДС, в настоящее время нет. По данным недавно опубликованного экспериментального исследования, выполненного на мышах, инфицированных паракокцидиомикозом, ранняя терапия пентоксифиллином и итраконазолом приводила к существенному уменьшению гранулематозного воспаления и редукции легочного фиброза [31].

Определенные перспективы в профилактике развития легочного фиброза может иметь ингаляционный гепарин. В недавно проведенном рандомизированном исследовании небулизированный гепарин у больных с ОДН, находящихся на ИВЛ, приводил к улучшению показателей оксигенации и уменьшению времени респираторной поддержки [32]. В данной работе были использованы довольно высокие дозы гепарина - по 25 тыс. единиц каждые 4-6 ч, до 14 дней. Несмотря на полученные обнадеживающие данные, пока сложно рекомендовать данную терапию в рутинной клинической практике - нет данных о возможности проведения терапии гепарином после ОПЛ / ОРДС, не отработаны оптимальные дозы препарата.

И наконец, еще одним перспективным направлением профилактики легочного фиброза после ОПЛ / ОРДС является антиоксидантная терапия. Оксидативное повреждение структур легких, т. е. повреждение, вызываемое активными формами кислорода или свободными радикалами, является одним из важных звеньев патогенеза ОПЛ / ОРДС [33-35]. Свободные радикалы вносят большой вклад в развитие интерстициального фиброза легких, т. к. вызывают повреждение и гибель клеток, изменяют и нарушают структуру и функцию клеточных и внеклеточных компонентов [36]. A.M.Cantin et al. показали, что низкий уровень одного из наиболее активных компонентов антиоксидантной защиты глютатиона (GSH) - способствует чрезмерной пролиферации фибробластов, что является ключевым событием аккумуляции экстрацеллюлярного матрикса при легочном фиброзе [37]. Более того, GSH регулирует активность одного из самых мощных фиброгенных цитокинов - трансформирующего фактора роста- $\beta$ [38]. Одним из лекарственных препаратов, способных увеличить эндогенный пул $\mathrm{GSH}$, является NAC. Он довольно легко проникает 
в клетки, где в результате реакции деацетилизации превращается в цистеин, т. е. является предшественником GSH.

В ряде исследований показано, что терапия NAC у больных ОПЛ / ОРДС приводит к быстрому и значительному улучшению показателей оксигенации, повышению сердечного выброса, к уменьшению длительности респираторной поддержки и времени пребывания больных в стационаре [39]. Кроме того, сегодня имеются основания для использования NAC и у больных с уже имеющимся фиброзом легких, например, при идиопатическом легочном фиброзе (ИЛФ). В пилотном открытом исследовании J.Behr et al. изучали эффективность в терапии NAC в дозе 1800 мг в сутки per os в течение 12 нед. у 18 больных ИЛФ [40]. К концу периода терапии было отмечено существенное повышение уровня глютатиона в жидкости бронхоальвеолярного лаважа $(p<0,005)$. Кроме того, авторы отметили положительную динамику функциональных легочных показателей (ЖЕЛ, $\mathrm{DL}_{\mathrm{CO}}$ и $\mathrm{PaO}_{2}$ при нагрузке). Очень обнадеживающими являются результаты рандомизированного контролируемого исследования IFIGENIA, в котором проводилось сравнение эффективности терапии NAC в дозе 1800 мг в сутки per os и стандартной терапии у 139 пациентов ИЛФ (все больные также получали преднизолон в дозе 0,5 мг / кг веса в сутки и азатиоприн в дозе 2 мг / кг веса в сутки) в течение 1 года [41]. На фоне терапии NAC у больных ИЛФ было отмечено замедление ухудшения функциональных показателей. Различие между группами через 12 мес. исследования по показателю ФЖЕЛ составило 0,18 л, или $9 \%(p=0,02)$, и по показателю $\mathrm{DL}_{\mathrm{Co}}-0,75$ ммоль / мин / кПа, или $24 \%(p=0,003)$. Таким образом, на основании этих данных в настоящем исследовании была назначена терапия NAC в высоких дозах (1 800 мг в сутки per os) больным в первые 3 мес. после перенесенного ОПЛ / ОРДС.

\section{Заключение}

Проведенная работа показала, что у пациентов, перенесших ОПЛ / ОРДС, вызванный вирусом гриппа A / H1N1, терапия NAC (Флуимуцил, Zambon Group, Италия) приводит к более быстрому улучшению показателей $\mathrm{DL}_{\mathrm{co}}$ и приросту пройденной дистанции в нагрузочном тесте. Интересно отметить, что достоверные различия были отмечены только на 3-м мес. наблюдения, в дальнейшем преимущество терапии NAC не было достоверным. Возможно, это отражает тот факт, что терапия NAC проводилась только в течение 3 мес., а после ее прекращения эффект со временем снизился. Необходимо проведение дальнейших исследований по изучению эффективности более длительного приема NAC после ОПЛ / ОРДС (например, 6 мес. и более).

\section{Литература}

1. Чучалин А.Г. Тяжелые формы гриппа: диагностические и лечебные алгоритмы. Пульмонология 2009; 5: 5-7.
2. Юшук Н.Д., Малышев Н.А., Авдеев С.Н. и др. Исходы тяжелого течения пандемического гриппа A / H1N1 / 2009. Tep. apx. 2010; 11: 15-18.

3. World Health Organization. Clinical management of human infection with pandemic (H1N1) 2009: revised guid-

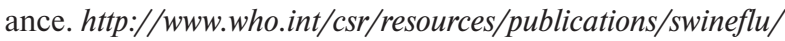
clinical management hln1.pdf

4. World Health Organization. Human infection with pandemic A (H1N1) 2009 influenza virus: clinical observations in hospitalized patients, Americas, July 2009 update. Wkly Epidemiol. Rec. 2009; 84: 305-308.

5. Авдеев С.Н. Пневмония и острый респираторный дистрес-синдром, вызванные вирусом гриппа А / H1N1. Пульмонология 2010; прил.: "Грипп А / H1N1: уроки пандемии": 32-46.

6. Perez-Padilla R., de la Rosa-Zamboni D., Ponce de Leon S. et al. Pneumonia and respiratory failure from swine-origin influenza A (H1N1) in Mexico. N. Engl. J. Med. 2009; 361: 680-689.

7. Jain S., Kamimoto L., Bramley A.M. et al. Hospitalized patients with 2009 H1N1 influenza in the United States, April-June 2009. N. Engl. J. Med. 2009; 361: 1935-1944.

8. Denholm J.T., Gordon C.L., Johnson P.D. et al. Hospitalised adult patients with pandemic (H1N1) 2009 influenza in Melbourne, Australia. Med. J. Aust. 2010; 192: 84-86.

9. Ware L.B., Matthay M.A. Acute respiratory distress syndrome. N. Engl. J. Med. 2000; 342: 1334-1349.

10. Herridge M.S., Cheung A.M., Tansey C.M. et al. One-year outcomes in survivors of the acute respiratory distress syndrome. N. Engl. J. Med. 2003; 348: 683-693.

11. Orme J., Romney J.S., Hopkins R.O. et al. Pulmonary function and health-related quality of life in survivors of acute respiratory distress syndrome. Am. J. Respir. Crit. Care Med. 2003; 167: 690-694.

12. Tansey C.M., Louie M., Loeb M. et al. One-year outcomes and health care utilization in survivors of severe acute respiratory syndrome. Arch. Intern. Med. 2007; 167: 1312-1320.

13. Hui D.S., Joynt G.M., Wong K.T. et al. Impact of severe acute respiratory syndrome (SARS) on pulmonary function, functional capacity and quality of life in a Hong Kong cohort of survivors. Thorax 2005; 60: 401-409.

14. Hui D.S., Wong K.T., Antonio G.E. et al. Long-term sequelae of SARS: physical, neuropsychiatric, and quality-of-life assessment. Hong Kong Med. J. 2009; 15 (Suppl. 8): $\mathrm{S} 21-\mathrm{S} 23$.

15. Bernard G.R., Artigas A., Brigham K.L. et al. The AmericanEuropean Consensus Conference on ARDS. Definitions, mechanisms, relevant outcomes, and clinical trial coordination. Am. J. Respir. Crit. Care Med. 1994; 149: 818-824.

16. Knaus W.A., Draper E.A., Wagner D.P., Zimmerman J.E. APACHE II: a severity of disease classification system. Crit. Care Med. 1985; 13: 818-829.

17. Marhsall J.C., Cook D.J., Christou N.V. et al. Multiple Organ Dysfunction Score: a reliable descriptor of a complex clinical outcome. Crit. Care Med. 1995; 23: 1638-1652.

18. Quanjer P.H., Tammeling G.J., Cotes J.E. Lung volumes and forced ventilatory flows. Report working party. Standardization of lung function tests. European Community for steel and coal. Official statement of the European Respiratory Society. Eur. Respir. J. 1993; 6: 5-40.

19. Cotes J.E., Chinn D.J., Quanjer P.H. Standardization of the measurement of transfer factor (Diffusing Capacity). Report working party, Standardization of lung function tests, European community for steel and coal. Official statement of the European Respiratory Society. Eur. Respir. J. 1993; 6: $41-52$. 
20. Mahler D., Weinberg D., Wells C., Feinstein A. The measurement of dyspnea: contents, interobserver agreement and physiologic correlates of two new clinical indexes. Chest 1984; 85: 751-758.

21. Borg G.A. Psychophysical basis of perceived exertion. Med. Sci. Sports Exerc. 1982; 14: 377-381.

22. Enright P.L., Sherrill D.L. Reference equations for the sixminute walk in healthy adults. Am. J. Respir. Crit. Care Med. 1998; 158: 1384-1387.

23. Davidson T.A., Caldwell E.S., Curtis J.R. et al. Reduced quality of life in survivors of acute respiratory distress syndrome compared with critically ill control patients. J.A.M.A. 1999; 281: 354-360.

24. Booth C.M., Matukas L.M., Tomlinson G.A. et al. Clinical features and short-term outcomes of 144 patients with SARS in the greater Toronto area. J.A.M.A. 2003; 289: 2801-2809.

25. Lee N., Hui D., Wu A. et al. A major outbreak of severe acute respiratory syndrome in Hong Kong. N. Engl. J. Med. 2003; 348: 1986-1994.

26. Hui D.S., Wong K.T., Ko F.W. et al. The 1-year impact of severe acute respiratory syndrome on pulmonary function, exercise capacity, and quality of life in a cohort of survivors. Chest 2005; 128: 2247-2261.

27. Ong K.C., Ng A.W., Lee L.S. et al. Pulmonary function and exercise capacity in survivors of severe acute respiratory syndrome. Eur. Respir. J. 2004; 24: 436-342.

28. Tsai L.K., Hsieh S.T., Chao C.C. et al. Neuromuscular disorders in severe acute respiratory syndrome. Arch. Neurol. 2004; 61: 1669-1673.

29. Herridge M.S., Tansey C.M., Matte A. et al. Functional disability 5 years after acute respiratory distress syndrome. N. Engl. J. Med. 2011; 364: 1293-1304.

30. Chang Y.-C., Yu C.-J., Chang S.-C. et al. Pulmonary sequelae in convalescent patients after severe acute respiratory syndrome: Evaluation with thin-section CT. Radiology 2005; 236: 1067-1075.

31. Naranjo T.W., Lopera D.E., Diaz-Granados L.R. et al. Combined itraconazole-pentoxifylline treatment promptly reduces lung fibrosis induced by chronic pulmonary paracoccidioidomycosis in mice. Pulm. Pharmacol. Ther. 2011; 24: 81-91.

32. Dixon B., Schultz M.J., Smith R. et al. Nebulized heparin is associated with fewer days of mechanical ventilation in critically ill patients: a randomized controlled trial. Crit. Care 2010, 14: R180.
33. Schraufstatter I.U., Revak S.D., Cochrane C.G. Proteases and oxidants in experimental pulmonary inflammatory injury. J. Clin. Invest. 1984; 73: 1175-1184.

34. Bunnell E., Pacht E.R. Oxidized glutathione is increased in the alveolar fluid of patients with the adult respiratory distress syndrome. Am. Rev. Respir. Dis. 1993; 148: 1174-1178.

35. Rahman I., MacNee $W$. Oxidative stress and regulation of glutathione in lung inflammation. Eur. Respir. J. 2000; 16: $534-554$

36. MacNee W., Rahman I. Oxidants / antioxidants in idiopathic pulmonary fibrosis. Thorax 1995; 50 (Suppl. 1): S53-S58.

37. Cantin A.M., Hubbard R.C., Crystal R.G. Glutathione deficiency in the epithelial lining fluid of the lower respiratory tract in idiopathic pulmonary fibrosis. Am. Rev. Respir. Dis. 1989; 139: 370-372.

38. Liu R.-M., Liu Y., Forman H.J. et al. Glutathione regulates transforming growth factor-b-stimulated collagen production of fibroblasts. Am. J. Physiol. Lung Cell. Mol. Physiol. 2004; 286: L121-L128.

39. Авдеев С.Н., Батын С.З., Мержоева З.М., Чучалин А.Г. Высокие дозы $\mathrm{N}$-ацетилцистеина при остром респираторном дистресс-синдроме. Вестн. анестезиол. и реаниматол. 2010; 5: 3-11.

40. Behr J., Maier K., Degenkolb B. et al. Antioxidative and clinical effects of high-dose $\mathrm{N}$-acetylcysteine in fibrosing alveolitis. Am. J. Respir. Crit. Care Med. 1997; 156: 1897-1901.

41. Demedts M., Behr J., Buhl R. et al. High-dose acetylcysteine in idiopathic pulmonary fibrosis. N. Engl. J. Med. 2005; 353: $2229-2242$

\section{Информация об авторах}

Авдеев Сергей Николаевич - д. м. Н., проф., руководитель клинического отдела ФГУ "НИИ пульмонологии" ФМБА России; тел. / Факс: (495) 465-52-64; e-mail: serg_avdeev@list.ru

Карчевская Наталья Анатольевна - научный сотрудник лаборатории интенсивной терапии и дыхательной недостаточности ФГУ "НИИ пульмонологии" ФМБА России; тел. / Факс: (495) 465-74-15; e-mail: karchevskaia@mail.ru

Баймаканова Гульсара Есенгельдиевна - к. м. н., старший научный сотрудник лаборатории интенсивной терапии и дыхательной недостаточности ФГУ "НИИ пульмонологии" ФМБА России; тел. / Факс: (495) 465-74-15; e-mail: gulsara.bai@mail.ru

Черняк Александр Владимирович - к. м. н., зав. лабораторией функциональных и ультразвуковых методов исследования ФГУ "НИИ пульмонологии" ФМБА России; тел. / факс: (495) 465-53-84; e-mail: achi2000@mail.ru 